\title{
Unit Verification: The CARA Experience ${ }^{\star}$
}

\author{
Arnab Ray, Rance Cleaveland \\ Department of Computer Science, SUNY at Stony Brook, Stony Brook, NY 11794-4400, USA
}

\begin{abstract}
The Computer-Aided Resuscitation Algorithm, or CARA, is part of a US Army-developed automated infusion device for treating blood loss experienced by combatants injured on the battlefield. CARA is responsible for automatically stabilizing a patient's blood pressure by infusing blood as needed, based on blood-pressure data the CARA system collects. The control part of the system is implemented in software, which is extremely safety-critical and thus must perform correctly.

This paper describes a case study in which a verification tool, the Concurrency Workbench of the New Century (CWB-NC), is used to analyze a model of the CARA system. The huge state space of the CARA makes it problematic to conduct traditional "push-button" automatic verification, such as model checking. Instead, we develop a technique, called unit verification, which entails taking small units of a system, putting them in a "verification harness" that exercises relevant executions appropriately within the unit, and then model checking these more tractable units. For systems, like CARA, whose requirements are localized to individual system components or interactions between small numbers of components, unit verification offers a means of coping with huge state spaces.
\end{abstract}

Key words: Model checking - state explosion - process algebra - abstraction - state minimization - formal methods

\section{Introduction}

The Computer-Assisted Resuscitation Algorithm (CARA) is a software system that provides closed-loop control to a high-

* Research supported by Army Research Office grants DAAD 190110003 and DAAD190110019, and by National Science Foundation grants CCR9988489 and CCR-0098037. The authors would also like to thank Dr. Fred Pearce and Mr. Steve Van Albert of the Walter Reed Army Institute for Research for allowing them to study the CARA system. output infusion pump [2]. Developed by researchers at the US Walter Reed Army Institute for Research (WRAIR), the system is intended to infuse fluids into patients who are in danger of developing hypotension due to blood loss sustained because of battlefield injuries. The system also has civilian applications in the treatment of shock trauma victims. In contrast with existing infusion systems, which require the constant attention of medical personnel, CARA is designed to operate automatically, thereby permitting a given number of medical personnel to attend to many more casualties. CARA is intended to be a component in the Life Support for Trauma and Transport (LSTAT) system, a state-of-the-art stretcher being developed with support from the US Army [1].

The fact that human lives depend on CARA makes it imperative that the software function correctly. At the same time, the complexity of the CARA system makes manual certification of the correctness of the system a difficult and expensive undertaking. In this paper we report on the use of an automatic formal verification tool, the Concurrency Workbench of the New Century (CWB-NC) [12-14], to analyze a model of CARA to determine whether it is consistent with requirements given for the system. While such an analysis does not guarantee that the deployed source code is correct, correct models are easier to turn into correct code than informal requirements are. At the same time, errors uncovered and eliminated at modeling time can be avoided at coding time, when they are much more difficult and expensive to fix.

Automatic verification tools provide users with, on the one hand, a modeling notation for systems, and, on the other, a notation for expressing system requirements. The tools then attempt automatically to determine whether a system model meets its requirements. The motivation for such tool development is to enable system designers to develop analyzable system artifacts early in the system-development process so that the ramifications of different design decisions, and errors and ambiguities in designer thinking, may be uncovered as soon as possible. Semantically, the modeling notations are based on different variants of finite-state machines. Requirements 
are often given either in temporal logic $[24,30]$ or also as state machines $[7,17,18,26]$. The term model checking [10] is often used to encompass algorithmic techniques for determining whether or not (formal) system models satisfy (formal) system requirements.

While model-checking tools have become very popular in the hardware community, their uptake in the field of software verification has been limited. One of the principal reasons for that is that for complex real-world systems, the semantic models (state machines) of the systems constructed for the purpose of analysis become so large that even powerful workstations cannot handle them. The problem is compounded when the system being modeled has real-time behavior, as the added obligation of tracking delays requires the introduction of even more states into these models. The resulting state explosion becomes even more dire when there are parallel modules whose behaviors must be interleaved.

As a real-time system possessing a number of concurrent components, CARA represents a difficult challenge to one interested in modeling and verification. In our modeling effort it quickly became obvious that no sufficiently detailed model of the the system could be verified using traditional "pushbutton" automatic verification, in which a user enters a model and a property and just "hits return." To cope with these challenges, we pursued an approach, which we call unit verification in analogy with the "unit testing" approach to software testing, for checking safety and liveness properties of models of software systems. Unit verification works by taking the property to be proved on the system and suitably crafting a "verification harness" based on that property. The "unit", or modules, inside the system to which the property is applicable is isolated, and all the behavior of the process not relevant to the property in question is "sealed" off. This transformed "unit" is then minimized and run inside the harness, which signals whether or not the property is satisfied by the system by engaging in pre-designated "good" or "bad" transitions.

The theoretical benefits of this approach are obvious. Huge state spaces become tractable because only the part of the state space relevant to the property in question is traversed; the uninteresting part of the system is abstracted away by "internalizing" the relevant state transitions. The conversion of external actions into internal actions also aids in minimizing the system to the furthest extent possible when verifying the property in question. This use of a targeted traversal of the state space leads to a dramatic reduction in the space needed to store the model.

Unit verification is most effective when the property being verified refers to a single module. The more modules the property spans (i.e. the more "global" it is), the less effective this approach is, due to state explosion. One's choice of module boundaries may thus be guided by the properties to be verified later on so that a majority of the properties pertain to a single module. For example, it might make sense to take two closely-coupled functional units and model it as a single module than as the parallel composition of two modules, so as to facilitate unit verification.
The paper is organized as follows. Section 2 gives an overview of the CARA system, while Section 3 introduces basic mathematical concepts related to modeling and verification and briefly discusses the tool used in the case study. Section 4 then describes the CARA system in more detail, while Section 5 presents our formal model of the system. The section following introduces unit verification and describes our experiences in using it to analyze the CARA model. Section 7 then discusses related work, while the last section states our conclusions and directions for future work.

\section{CARA System Overview}

The system under study is known as the CARA (ComputerAided Resuscitation Algorithm) control software [3-5], which is being developed in the context of the CARA infusion-pump project sponsored by the Walter Reed Army Institute of Research (WRAIR) in collaboration with the Food and Drug Administration (FDA). The goal of this project is to develop a device that automatically infuses fluids into a trauma patient as necessary in order to stabilize the patient's blood pressure.

The information about CARA contained in this paper is taken from three documents provided by WRAIR researchers. These include a requirements document containing a numbered list of 148 requirements [4]; a question and answer document regarding these requirements [5]; and a hazards analysis document [3]. Additional clarifications on the system were obtained from WRAIR personnel.

\subsection{Background}

CARA comprises software for monitoring and controlling the operation of an M100 infusion pump, which is a device that drives resuscitating fluids into an injured patient's bloodstream. The system is designed for use in a battlefield situation and is intended to assist a care-giver by automatically monitoring the patient's blood pressure and delivering fluid as required to maintain a pre-selected blood pressure. Besides controlling the pump, CARA also logs the patient's condition and provides diagnostic information in case problems arise with either the patient or the machine. So the CARA software's chief responsibilities are to:

1. continually monitor and $\log$ the blood pressure of the patient;

2. use the blood pressure information to determine the control voltage to be applied to the pump to maintain a suitable rate of infusion; and

3. sound alarms and provide diagnostic information in case of any sudden change in the patient's condition or malfunction of the infusion pump.

CARA is intended to increase the number of patients a given number of medical personnel may care for. In a traditional resuscitation setting, injured patients are connected to an infusion pump whose behavior is governed by different hardware settings on the pump itself. These settings must 


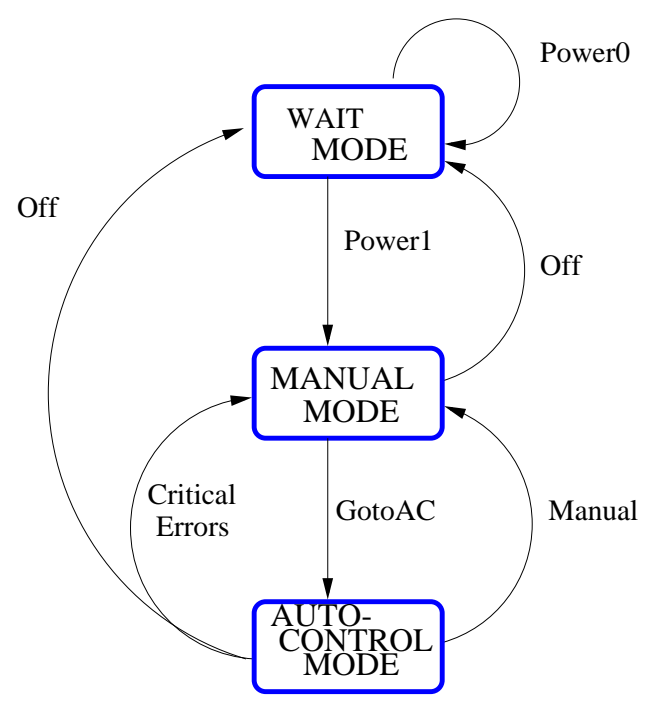

Fig. 1. CARA Reference Model: Main Modes

be closely monitored by a care-giver, who continually adjusts them depending on the condition of the patient and takes action if a system malfunction occurs. In a battlefield setting, when one care-giver may be attending many casualties at one time, medical manpower is often insufficient, and patients suffer debilitating and often fatal consequences relating to inadequately monitored infusion equipment. CARA represents a way for automating the work of a care-giver so that the infusion process can function with minimum human intervention.

\subsection{Modes of Operation}

The remainder of this section describes how the CARA software achieves the aims just mentioned. The system has three main modes of operation: Wait mode, Manual mode, and Autocontrol mode. Fig. 1 summarizes how the modes interact; each mode is described below.

\subsubsection{Wait Mode}

The system is in this mode when the pump is off: it performs no monitoring or pump control.

\subsubsection{Manual Mode}

The software enters manual mode when the pump is initially turned on. When the system is in this state the software only performs monitoring functions; it does not send control signals to the pump, which instead uses default hardware settings set by the care-giver to drive the infusion rate. There are several anomalous conditions constantly being monitored in this mode, however: whether there is an air bubble inside the tube, whether the tube through which fluid is being pumped is free from leakage, and whether the pump is in proper working order. There is a 5-second polling cycle for these conditions along with a specified polling order. An error triggers several alarms based upon how critical the associated condition is. If the power supply to the pump is lost, the control goes to a backup battery, and a high-priority alarm is sounded.

There are two ways to leave manual mode: the pump may be turned off, or the care-giver may press the auto-control mode button to transfer the software to auto-control mode. This button is only enabled when the pump is in its normal operative mode (i.e. no error conditions are present); the button that initiates this mode is disabled otherwise.

\subsubsection{Auto-Control Mode}

In auto-control mode, CARA assumes two roles: monitoring the status lines from the pump as well as controlling the infusion rate. In this mode it also supplies diagnostic information to the care-giver via a display screen in case of exceptions and maintains a log file of errors, trend data and other data that would ordinarily be collected by the care-giver. When the CARA system is in auto-control mode, the care giver plays a much less active role, and when intervention is required the software provides suggestions on how to proceed.

At the heart of the CARA system is a PID control algorithm that takes as inputs the current and desired blood pressures of the patient and, based on the difference between these, adjusts the voltage driving the infusion pump.

CARA is designed to use up to three sources of blood pressure data: an arterial line, pulse-wave transmission and a cuff. Each of these sources can be used as input to the PID control algorithm. Since these data sources may be simultaneously available the system uses a priority scheme to determine which source to use: an arterial line has highest priority, followed by pulse-wave transmissions and a cuff, in that order. Thus, if all three sources are available the arterial line is used as the source of the patient's blood pressure. If the arterial line source is lost then the pulse-wave source is used, and if that is also lost, then the cuff source is used. 
For reasons of patient safety the CARA also checks the integrity of the blood-pressure data it collects. This "corroboration process" involves checking values delivered by either the arterial or pulse wave to those obtained via the cuff. If the blood pressures are within an acceptable range of difference, they are said to be corroborated, else they are are not corroborated. If an available source does not corroborate with the cuff pressure, then the care-giver is prompted and presented with the option of overriding and using the uncorroborated source for the control algorithm. If the care-giver does not want to override then the next priority source is sought to be corroborated. If that too cannot be corroborated then the software proceeds using the cuff pressure as the control pressure.

Once a blood-pressure source has been selected the data it collects is used as input to the PID control algorithm. This algorithm checks whether the current blood pressure is below the target value or not. If it is below then it sets an appropriate pump-control voltage. If the target blood pressure value has been attained or exceeded then the control voltage is set to zero, meaning that the infusion ceases.

The care-giver can reset the target blood-pressure value by entering new input parameters, after which the PID algorithm restarts. But this entering of new values cannot be done unless all the components are working properly: any error prevents the care-giver from entering new input parameters.

Re-corroboration of blood pressure sources takes place every 30 minutes, except that when a new source becomes available that has a higher priority than the source currently being used, corroboration of the new source is attempted immediately. Corroboration is also stalled when an override question is pending. Once corroborated, a source will continue to be used until the next re-corroboration cycle or until a higherpriority source becomes corroborated. All sources are monitored continually, and appropriate action is taken immediately in case a source is lost. Thus, while a care-giver may have to wait up to 30 minutes to detect that a corroborated source has become uncorroborated, an immediate action (alarm and state change) occurs if a corroborated source is lost.

When the blood pressure of all sources becomes zero, alarms are sounded, and after waiting for specified periods of time the software goes back to manual mode. A care-giver can also return the system to manual mode by pressing the appropriate button.

\section{Modeling Preliminaries}

In this section we describe the basic mathematical machinery used in our modeling and analysis of the CARA system. Before discussing the theory, however, we note that the following characteristics are important in the selection of an appropriate framework.

Real time. The CARA system includes a number of timing constraints. To be maximally useful, a modeling notation should include support for these.
Component interaction. The CARA system includes many components that interact either directly with one another or with the environment. To model CARA effectively, a modeling notation needs to support a flexible notion of component interaction.

Subsystem analysis. To cope with state explosion our unitverification approach requires being able to isolate subsystems within a larger system. An appropriate modeling notation should therefore make it easy to treat system modules independently.

\subsection{Discrete-Time Labeled Transition Systems}

The basic semantic framework used in our modeling is discretetime labeled transition systems. To define these we first introduce the following.

Definition 1. A set $A$ is a set of visible actions if is is nonempty and does not contain $\tau$ or 1 .

In what follows visible-action sets will correspond to the atomic interactions users will employ to build system models. The distinguished elements $\tau$ and 1 correspond to the internal action and clock-tick (or idling) action. For notational convenience, given a visible-action set $A$ we define:

$$
\begin{aligned}
A_{\{\tau\}} & =A \cup\{\tau\} \\
A_{\{1\}} & =A \cup\{1\} \\
A_{\{\tau, 1\}} & =A \cup\{\tau, 1\}
\end{aligned}
$$

We sometimes call the set $A_{\{\tau, 1\}}$ an action set and $A_{\{1\}}$ as a controllable-action set (the reason for the latter being that in many settings, actions in this set can be "controlled" to a certain extent by a system environment).

Discrete-time labeled transition systems are defined as follows.

Definition 2. A discrete-time labeled transition system (DTLTS) is a tuple $\left\langle S, A, \rightarrow, s_{I}\right\rangle$ where:

1. $S$ is a set of states;

2. $A$ is a visible-action set (cf. Def. 1);

3. $\longrightarrow \subseteq S \times A_{\{\tau, 1\}} \times S$ is the transition relation, and 4. $s_{I} \in S$ is the start state.

A DTLTS $\left\langle S, A, \rightarrow, s_{I}\right\rangle$ satisfies the maximal-progress property if for every $s$ such that $s \stackrel{\tau}{\longrightarrow} s^{\prime}$ for some $s^{\prime}, s \stackrel{1}{\longrightarrow} s^{\prime \prime}$ for any $s^{\prime \prime}$.

A DTLTS $\left\langle S, A, \rightarrow, s_{I}\right\rangle$ encodes the operational behavior of a real-time system. States may be seen as "configurations" the system may enter, while actions represent interactions with the system's environment that can cause state changes. The transition relation records which state changes may occur: if $\left\langle s, a, s^{\prime}\right\rangle \in \longrightarrow$ then a transition from state $s$ to $s^{\prime}$ may take place whenever action $a$ is enabled. Generally speaking, $\tau$ is always enabled; other actions may require "permission" from the environment in order to be enabled. Also, transitions except those labeled by 1 are assumed to be instantaneous. 
While unrealistic at a certain level, this assumption is mathematically convenient, and realistic systems, in which all transitions "take time", can be easily modeled. We write $s \stackrel{a}{\longrightarrow} s^{\prime}$ when a system in state $s$ transitions, via action $a$, to state $s^{\prime}$.

If a DTLTS satisfying the maximal progress property is in a state in which internal computation is possible, then no idling (clock ticks) can occur.

DTLTSs model the passage of time and interactions with a system's environment. Discrete-time process algebras such as Temporal CCS [27] enrich the basic theory of DTLTSs with operators for composing individual DTLTSs into systems that may themselves be interpreted via (global) DTLTSs. Such languages typically include operators for parallel composition and action scoping, among others. The variant of Temporal CCS used in this paper, for instance, may be defined as follows. Let $\Lambda$ be a nonempty set of labels not containing $\tau$ and 1 , and fix $A^{\text {TCCS }}=\Lambda \cup\{\bar{\lambda} \mid \lambda \in \Lambda\}$, where ${ }^{-}$ is a syntactic operator. Intuitively, $\Lambda$ contains the set of communication channels, with visible Temporal CCS actions of the form $\lambda$ corresponding to receive actions on port $\lambda$ and $\bar{\lambda}$ corresponding to send actions on port $\lambda$. Then (a subset of) Temporal CCS is the set of terms defined by the following grammar, where $L \subseteq \Lambda$ and $M$ is a maximal-progress DTLTS whose action set is $A^{\mathrm{TCCS}}$.

$$
P::=M\left|P_{1}\right| P_{2} \mid P \backslash L
$$

Intuitively, these constructs may be understood in terms of the communication actions and units of delay (or idling) they may engage in. $P_{1} \mid P_{2}$ represents the parallel composition of $P_{1}$ and $P_{2}$. For the composite system to idle, both components must be capable of idling. Non-delay transitions are executed in an interleaved fashion; moreover, if either $P_{1}$ or $P_{2}$ is capable of an output $(\bar{\lambda})$ on a channel $\lambda$ that the other is capable of an input on $(\lambda)$, then a synchronization occurs, with both processes performing their actions and a $\tau$ resulting: in this case, no idling is possible until after the $\tau$ is performed. If $L \subseteq A$ then $P \backslash L$ defines a process in which the channels or actions in $L$ may be thought of as "local". In other words, actions involving the channels in the set $L$ are prevented from interacting with the outside environment. The net effect is to "clip", or remove, transitions labeled by such actions from $P$. Other operators, including a hiding operator $P\lfloor L\rfloor$ that converts actions whose labels are in $L$ into $\tau$ actions, may be defined in terms of these.

This informal account may be formalized by giving rules for converting Temporal CCS terms into DTLTSs in the standard Structural Operational Style [29].

Finally, DTLTSs may be minimized by merging semantically equivalent but distinct states. In this paper a specific equivalence, Milner's observational equivalence [26], is used for this purpose. Intuitively, two states in a DTLTS are observationally equivalent if, whenever one is capable of a transition to a new state, then the other is capable of a sequence of transitions with the same "visible content" to a state that is observationally equivalent to the new state. To define observational equivalence precisely, we use the following notions.
Definition 3. Let $M=\left\langle S, A, \rightarrow, s_{I}\right\rangle$ be a DTLTS, with $s, s^{\prime} \in S$ and $a \in A_{\{\tau, 1\}}$.

1. $s \stackrel{\epsilon}{\Longrightarrow} s^{\prime}$ if there exists $s=s_{0}, \ldots s_{n}=s^{\prime}$ such that for all $0 \leq i<n, s_{i} \stackrel{\tau}{\rightarrow} s_{i+1}$.

2. $s \stackrel{a}{\longrightarrow} s^{\prime}$ if there exists $s_{1}, s_{2}$ such that $s \stackrel{\epsilon}{\longrightarrow} s_{1} \stackrel{a}{\longrightarrow}$ $s_{2} \stackrel{\epsilon}{\Longrightarrow} s^{\prime}$.

3. The visible content, $\hat{a}$, of $a$ is defined by: $\hat{\tau}=\epsilon$ and $\hat{a}=a$ if $a \neq \tau$.

4. A relation $R \subseteq S \times S$ is a weak bisimulation if, for every $a \in A_{\{\tau, 1\}}$ and $\left\langle s_{1}, s_{2}\right\rangle \in R$, the following hold.

(a) If $s_{1} \stackrel{a}{\longrightarrow} s_{1}^{\prime}$ then there exists $s_{2}^{\prime}$ such that $s_{2} \stackrel{\hat{a}}{\Longrightarrow} s_{2}^{\prime}$ and $\left\langle s_{1}^{\prime}, s_{2}^{\prime}\right\rangle \in R$.

(b) If $s_{2} \stackrel{a}{\longrightarrow} s_{2}^{\prime}$ then there exists $s_{1}^{\prime}$ such that $s_{1} \stackrel{\hat{a}}{\Longrightarrow} s_{1}^{\prime}$ and $\left\langle s_{1}^{\prime}, s_{2}^{\prime}\right\rangle \in R$.

5. $s_{1}$ and $s_{2}$ are observationally equivalent, written $s_{1} \approx s_{2}$, if there exists a weak bisimulation $R$ with $\left\langle s_{1}, s_{2}\right\rangle \in R$.

Intuitively, $s \stackrel{\epsilon}{\Longrightarrow} s^{\prime}$ if there is a sequence of internal transitions leading from $s$ to $s^{\prime}$, while $s \stackrel{a}{\Longrightarrow} s^{\prime}$ if there is a sequence of transitions, one labeled by $a$ and the rest by $\tau$, leading from $s$ to $s^{\prime}$. The visible content of $\tau$ is "empty" $(\epsilon)$.

It can be shown that observational equivalence is indeed an equivalence relation on states, and that observationally equivalent states in a DTLTS can be merged into single states without affecting the semantics of the over-all DTLTS. ${ }^{1}$ It is also the case that, in the context of the Temporal CCS operators mentioned above, DTLTSs may be freely replaced by their minimized counterparts without affecting the semantics of the overall system description. For finite-state DTLTSs, polynomial-time algorithms for minimizing DTLTSs with respect to observational equivalence have been developed [12, $16,19,28]$. This concept will be used later when defining the minimization procedure for unit verification.

\subsection{Model Checking}

In automated model-checking approaches to system verification system properties are formulated in a temporal logic; the model checker then determines whether or not they hold of a given (finite-state) system description. A given temporallogic formula defines the behavior a system should exhibit as it executes; as such, temporal logic extends more familiar notations such as the propositional calculus with operators enabling one to describe how a system behaves as time passes.

In this work we use a (very small) subset of the modal mu-calculus [21], a temporal logic for describing properties of (discrete-time) labeled transition systems. The syntax of the fragment is described as follows, where $A$ is a visibleaction set (cf. Def. 1).

$$
\phi::=\mathrm{tt}|\mathrm{ff}|\langle a\rangle_{\{\tau\}} \phi\left|[a]_{\{\tau\}} \phi\right|\langle a\rangle_{\{\tau, 1\}} \phi \mid[a]_{\{\tau, 1\}} \phi
$$

${ }^{1}$ More precisely, the notion of observational equivalence can be lifted to a relation between DTLTSs, rather than just between states in the same DTLTS. It can then be shown that a DTLTS is observationally equivalent to its minimized counterpart. 
Here $a \in A_{\{1\}} \cup\{\epsilon\}$. The full mu-calculus contains other operators, including conjunction, disjunction and recursion constructs; a full account may be found in [21].

These formulas are interpreted with respect states in a given DTLTS. The formulas tt and ff represent the constants "true" and "false" and hold of all, respectively no, states. The remaining operators are modal in that they refer to the transition behavior of a state. In particular, a state $s$ satisfies $\langle a\rangle_{\{\tau\}} \phi$ if there is another state $s^{\prime}$ such that $s \stackrel{a}{\Rightarrow} s^{\prime}$ and $s^{\prime}$ satisfies $\phi$, while $s$ satisfies $[a]_{\{\tau\}} \phi$ if every $s^{\prime}$ such that $s \stackrel{a}{\Longrightarrow} s^{\prime}$ satisfies $\phi$. The operators $\langle a\rangle_{\{\tau, 1\}}$ and $[a]_{\{\tau, 1\}}$ are similar except that they treat clock ticks as being analogous to $\tau$-transitions. More precisely, we define the following.

Definition 4. Let $M=\left\langle S, A, \longrightarrow, s_{I}\right\rangle$ be a DTLTS, with $s, s^{\prime} \in S$ and $a \in A_{\{\tau, 1\}}$.

1. $s \stackrel{\epsilon}{\Rightarrow} s^{\prime}$ if there exists $s=s_{0}, \ldots, s_{n}=s^{\prime}(n \geq 0)$ and $a_{1}, \ldots a_{n}$ such that $s_{0} \stackrel{a_{1}}{\longrightarrow} s_{1} \cdots s_{n-1} \stackrel{a_{n}}{\longrightarrow} s_{n}$ and $a_{i} \in\{\tau, 1\}$ for all $1 \leq i \leq n$.

2. $s \stackrel{a}{\Rightarrow} s^{\prime}$ if there exists $s_{1}, s_{2}$ such that $s \stackrel{\epsilon}{\Rightarrow} s_{1} \stackrel{a}{\longrightarrow} s_{2} \stackrel{\epsilon}{\Rightarrow}$ $s^{\prime}$.

So $s \stackrel{\epsilon}{\Rightarrow} s^{\prime}$ if there is a sequence of $\tau-$ and $1-$ transitions leading from $s$ to $s^{\prime}$, while $s \stackrel{a}{\Rightarrow} s^{\prime}$ if there is a sequence of transitions, one labeled by $a$ and the rest either by $\tau$ or 1 , leading from $s$ to $s^{\prime}$.

We can now define $\langle a\rangle_{\{\tau, 1\}}$ and $[a]_{\{\tau, 1\}}$ more precisely. A state $s$ satisfies $\langle a\rangle_{\{\tau, 1\}} \phi$ if there is an $s^{\prime}$ such that $s \stackrel{a}{\Rightarrow} s^{\prime}$ and $s^{\prime}$ satisfies $\phi$. Dually, $s$ satisfies $[a]_{\{\tau, 1\}} \phi$ if every $s^{\prime}$ reachable via a $\stackrel{a}{\Rightarrow}$ transition from $s$ satisfies $\phi$.

The operators \langle\rangle$_{\{\tau\}},[]_{\{\tau\}},\langle\rangle_{\{\tau, 1\}}$ and []$_{\{\tau, 1\}}$ are not primitive mu-calculus operators, but they can be encoded using the primitive operators.

In what follows we write $M \models \phi$ if $M$ is a DTLTS whose start state satisfies $\phi$.

\subsection{The Concurrency Workbench of the New Century}

In the case study we use the Concurrency Workbench of the New Century (CWB-NC) [12-14] as the verification engine for conducting our analysis of CARA.

The CWB-NC supports several different types of verification, including mu-calculus modeling checking, various kinds of refinement checking, and several types of semantic equivalence checking. The tool also includes routines for minimizing systems with respect to different semantic equivalences, including observational equivalence.

The design of the CWB-NC makes it relatively easy to retarget it to different design languages. The Process Algebra Compiler (PAC) tool $[11,14]$ provides support for adapting the design language processed by the CWB-NC. In the case of CARA, we started with a basic Temporal CCS CWB-NC front end included in the release of the tool and modified it slightly to include constructs, such as the disabling construct from LOTOS [8], that simplified the modeling of the system.

\section{A CARA Reference Model}

In order to develop formal models of CARA suitable for analysis by the CWB-NC we first define a reference model for the system. This model has two components.

Modes. A high-level rendering of the modes the software can be in. CARA's modes are described in Section 2.2 and Fig. 1.

Architecture. A decomposition of the system into communicating components, each of which is modeled operationally using finite-state machines.

The architectural component of the reference model is given in Fig. 2, which also provides an abbreviated description of the interactions between the modules in the architecture. In this diagram ovals represent system components, while circles constitute environment components.

The remainder of this section provides a brief description of each component in the CARA architecture. Before giving this, however, we first note that none of the CARA documents explicate the system architecture; we have instead devised one based on the rationale that there should be one module for each physical component or major control unit of the system. Care was also taken to minimize the communication interfaces between components so that components were as independent of each other as possible. The interprocess communication, though not explicitly stated in the design documents, was assumed to be via synchronous message passing or through shared variables.

\subsection{Alarm}

This Alarm module is modeled as a system that takes in two types of error conditions, HighAlarm or LowAlarm. Depending on what type of an alarm it is, the alarm determines its "silencing time," that is, the amount of time that it will be silenced when a care-giver presses the Silence Alarm button. Note that the Alarm's audible and visible indicators are completely deactivated only when all the conditions that caused an alarm to be raised have been fixed.

\subsection{Alarm Control}

The Alarm module deals with the hardware component of the visual and audible alarms. The decision as to when the alarm module is to be set or reset is handled by the Alarm Control. As the name suggests, this is the controller process for the Alarm. It takes as its input all possible alarm conditions from all possible modules that can raise an alarm and then, based on its internal logic, decides whether to raise a high or a low alarm. This architecture makes it possible to make the alarm-control logic independent of the actual hardware modeling of the alarm. Thus, even if in the future the logic for alarm-control changes, only this part of the system needs to be changed. This paradigm of separating the physical device from its controller is a principle we have followed in the entire reference-model design. 


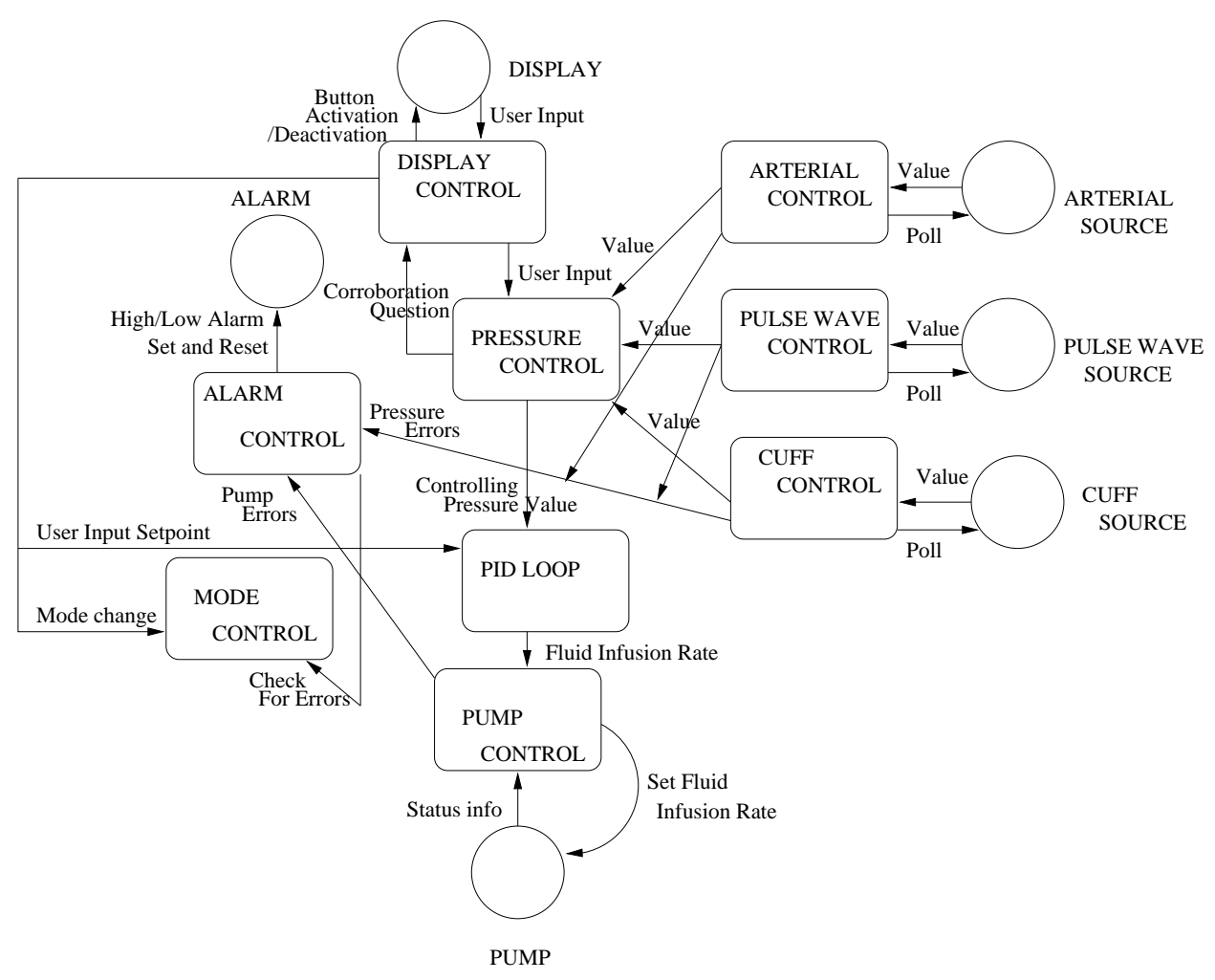

Fig. 2. CARA Reference Model: System Architecture and Module Interaction.

\subsection{Pump}

The Pump module is the physical device that pumps fluids into the patient. In our model the pump is modeled as a black box: since the internal workings of the pump is outside the scope of the design documents that were supplied, the pump is taken to be a monolithic entity which only supplies data on the pump-status lines. In other words, the pump is treated as a source of data to the rest of the system, and nothing else.

\subsection{Pump Control}

The pump status is communicated to CARA in two ways: either through interrupts (continuity, occlusion, power) or through polling (air, emf, impedance). The Pump Control's functions are to monitor the interrupts continually, so that action may be taken when they come, and to monitor the poll lines according to a given frequency. The Pump Control is also responsible for determining when to raise an alarm and for conducting subsidiary checks when an error occurs (e.g. whenever an emf-error occurs the impedance is also checked).

The Pump Control also takes input from the PID Algorithm and changes the hardware settings of the pump so that it can pump at the requisite rate. The control outputs are treated as "visible actions" that are offered to the environment of the CARA model. This is because we do not model the physical workings of the Pump and thus cannot simulate Pump behavior in response to a given control signal.

\subsection{Display}

The Display module consists of the interface presented to the care-giver in order to control the CARA system. It comprises buttons that enable the care-giver to make mode changes, input new target blood-pressure values, or resolve corroboration questions regarding whether or not an uncorroborated blood pressure is to be overridden.

\subsection{Display Control}

Display buttons are not always available to a care-giver. For example, the system can only enter auto-control mode when there are no error conditions in the system. Hence, if there is an error anywhere in the system the Start Auto-control button should be "grayed out." Similarly there is a priority to the input windows that are offered to the user when multiple user inputs are needed. For example, a corroboration window would have a higher priority than the new input parameter window. Maintaining the priority information and suitably activating/de-activating buttons is the job of the Display Control.

\subsection{Mode Control}

There are two ways of affecting a change in mode within CARA. One is when the user engages in explicit button presses 
on the Display. This aspect is dealt with in the Display Control module. But there are other ways of changing mode. In auto-control mode, for example, there are several error conditions which, if persistent for specific periods of time, necessitate a change to manual mode. This autonomous mode change is handled by the Mode Control.

In addition, there are error conditions to be signaled if a required blood-pressure range is not attained within a specific time after auto-control initiation. Mode Control also keeps track of the time instant at which auto-control mode was entered.

\subsection{Sources}

There three different Sources modules, one for each potential source of blood-pressure readings: Arterial, Pulse-Wave and Cuff. These are basically stub processes that model potential patient behavior.

\subsection{Source Control}

The Source Control modules are also three different, independent modules, one for each potential source. Source Control primarily deals with the frequency of polling the respective source being controlled. It also deals with the issue of when to signal errors or, more specifically, how many poll failures are required before an error is flagged. It additionally supplies the eventual blood-pressure value to the Pressure Control module, and this value is used for the PID loop.

\subsection{Pressure Control}

This control module may be considered to be the most complex module in the system. Its first function is to determine which blood-pressure source to use as the controlling source. It compares blood pressures from different sources to corroborate them. It keeps track of when to corroborate the pressure sources. If a blood-pressure source becomes uncorroborated, it signals the Display module to ask the override question and takes action according to the user supplied input. If a higher priority blood pressure than the current controlling blood pressure starts reporting valid values and no override is pending, it immediately takes action.

\subsection{PID Loop}

This module compares the controlling blood pressure value to the user-set set-point value and controls the fluid-infusion rate on the basis of whether the set-point has been attained or not.

\section{Modeling CARA in Temporal CCS}

To model CARA so that it can be analyzed in the CWB-NC, we first must encode the reference model described in the previous sections in the version of Temporal CCS supported by the tool. This section describes this encoding.

Our general modeling strategy is to "implement" each module in the reference model as a DTLTS and then interconnect these DTLTSs using the other operators from Temporal CCS. In practice, because the Temporal CCS model must concern itself with implementation details (e.g. how shared variables are represented) that the reference model does not, we used several DTLTSs for each reference module. For instance, the Temporal CCS model contains 23 different individual DTLTSs to implement the 23 shared variables (21 boolean-valued, one eight-valued, and one nine-valued) used to exchange data between the other modules. Table 1 lists the DTLTSs in our Temporal CCS model, together with a brief discussion of what behavior each DTLTS is responsible for.

Figs. 3 and 4 give example DTLTSs taken from our model. In the case of the CuffControl module, what is shown is the minimized version of the DTLTS; to simplify the diagram, we have also omitted the clock-tick transitions (every state has a clock-tick transition back to itself in this case). This DTLTS encodes the following behavior. When instructed to take a cuff reading, the cuff control executes an action to get a cuff value. If the value is valid, then this is recorded, and any alarm due to a lost cuff is disabled. If the value is invalid, then another cuff reading is attempted. If the second value is valid, then the previous sequence of events is repeated; otherwise, the cuff is determined to be invalid, and an alarm raised.

The BPMonitor DTLTS is larger than CuffControl, since the module it models is more complex. For clarity a number of transitions, including clock-tick transitions that lead back to the state from which they originate, have been left out. In addition, sequences of clock-tick transitions have been collapsed into single transitions labeled by the number of clock ticks.

The Temporal CCS implementation of the Alarm module consists of three separate DTLTSs: one for a high-priority alarm, one for a low-priority alarm, and a controller that activates and deactivates the alarms as appropriate. These DTLTSs are named HighRinger, LowRinger, and AlarmController, respectively. The Temporal CCS expression Alarm is then:

(HighRinger | LowRinger | AlarmController) \L

where $L$ contains the labels of the actions used by AlarmController to activate and deactivate the alarms.

Table 2 contains size data for each of the Temporal CCS DTLTSs given above. The second and third columns list the number of states and transitions for each of these DTLTSs; the next two give the sizes after the system have been minimized with respect to observational equivalence. The final column gives the amount of CPU time needed to perform the minimization within the CWB-NC. All the experiments were carried out on a Sun workstation running Solaris 2.6, with a $360 \mathrm{MHz}$ UltraSparc II processor, $256 \mathrm{MB}$ of RAM, and 1 GB of swap space.

The sizes of these individual DTLTSs imply that the entire CARA system contains in excess of $1.85 \cdot 10^{40}$ states. 


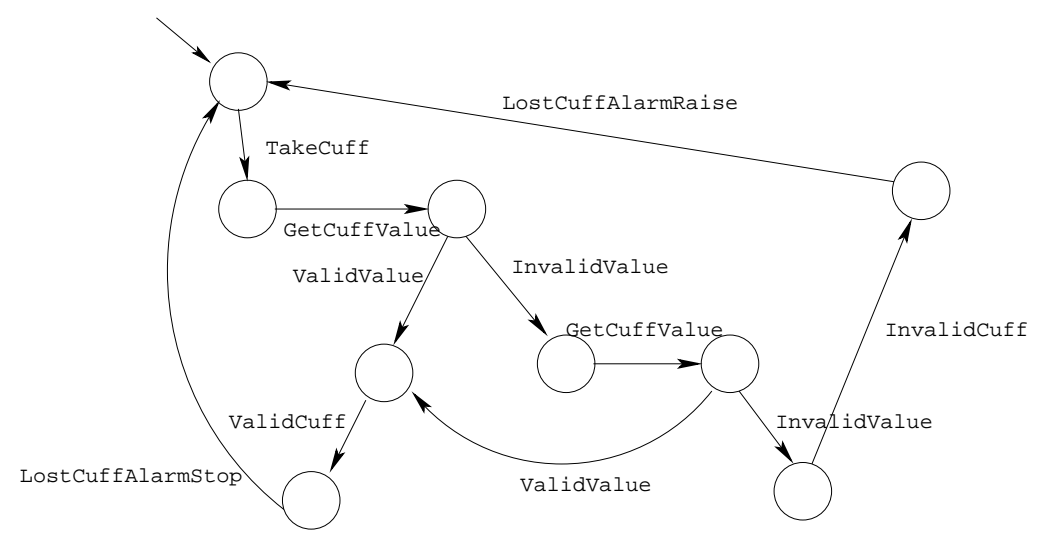

Fig. 3. The CuffControl Module.

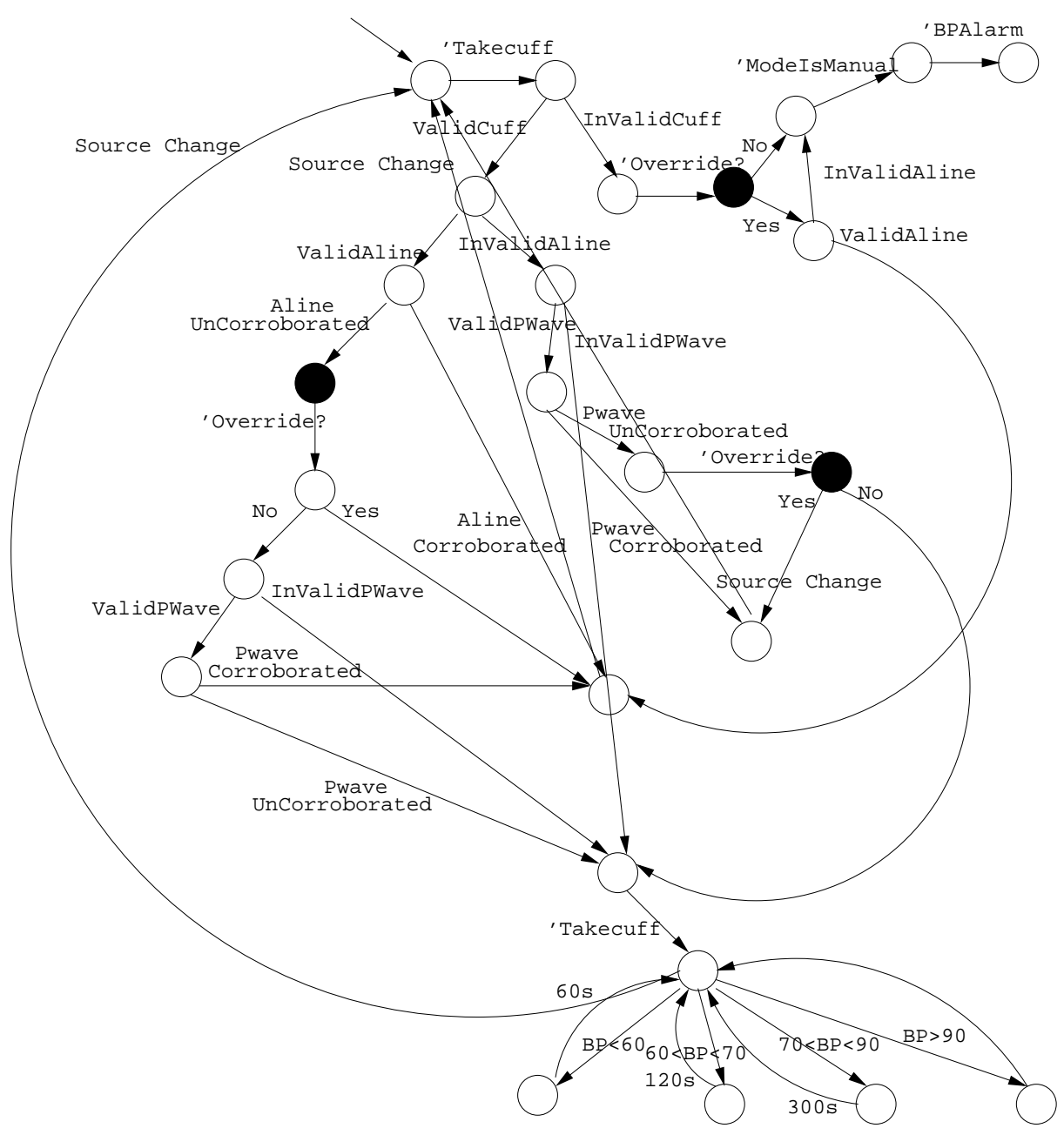

Fig. 4. The BPMonitor Module. For clarity, some transitions have been omitted. White states have a transition to the start state upon the receipt of a corroboration signal; black states do not. 
Table 1. Modules in the Temporal CCS Model of CARA.

\begin{tabular}{|l|l|}
\hline Module & Purpose \\
\hline Alarm & Raises alarms \\
CorrobControl & Controls when corroboration takes place \\
AlarmControl & Parses the different errors and signals the Alarm \\
AirChecker & Checks the Air \\
EmfChecker & Checks the Emf \\
ContinuityChecker & Checks Continuity \\
OcclusionChecker & Checks Occlusion \\
PumpPowerChecker & Checks Power \\
Display & Handles user input and window priorities \\
OverrideControl & Controls the Override question \\
ModeControl & Controls when mode change takes place \\
PressureControl & Checks to see if desired pressure is attained within a certain time after infusion started \\
PIDControl & Handles the PID Loop \\
PWaveControl & Controls the acquisition of pulse wave \\
AlineControl & Controls the acquisition of arterial line \\
CuffControl & Controls the acquisition of cuff when all other sources are lost \\
BPMonitor & Controls priority among different pressure sources and determines the controlling pressure source \\
BPDropMonitor & Checks to see if there is a blood pressure drop after attainment of steady value \\
Misc. variables & Shared variables used for inter-module communication \\
\hline
\end{tabular}

Table 2. Size Data for Modules in Table 1.

\begin{tabular}{|l|r|r|r|r|r|}
\hline \multirow{2}{*}{ Module } & \multicolumn{2}{|c|}{ Original Size } & \multicolumn{2}{c|}{ Minimized size } & Minimization \\
\cline { 2 - 5 } & States & Transitions & States & Transitions & Time (sec.) \\
\hline Alarm & 104 & 452 & 19 & 76 & 0.600 \\
CorrobControl & 1,805 & 3,619 & 1,804 & 3,613 & 3.030 \\
AlarmControl & 6 & 66 & 6 & 66 & 0.820 \\
AirChecker & 893 & 2,504 & 225 & 606 & 2.440 \\
EmfChecker & 555 & 1,477 & 345 & 968 & 1.560 \\
ContinuityChecker & 6 & 18 & 4 & 12 & 0.010 \\
OcclusionChecker & 6 & 18 & 4 & 12 & 0.010 \\
PumpPowerChecker & 6 & 18 & 4 & 12 & 0.010 \\
Display & 68 & 343 & 44 & 205 & 0.360 \\
OverrideControl & 9 & 54 & 5 & 30 & 0.070 \\
ModeControl & 912 & 5,172 & 606 & 3,028 & 5.730 \\
PressureControl & 4 & 11 & 4 & 11 & 0.000 \\
PIDControl & 1,206 & 1,214 & 1,205 & 1,211 & 0.830 \\
PWaveControl & 163 & 209 & 153 & 180 & 0.180 \\
AlineControl & 163 & 209 & 153 & 180 & 0.120 \\
CuffControl & 11 & 26 & 9 & 20 & 0.010 \\
BPMonitor & 646 & 748 & 627 & 683 & 0.540 \\
BPDropMonitor & 18 & 66 & 13 & 41 & 0.050 \\
Misc. variables & $\sim 1.44 \cdot 10^{8}$ & $\sim 1.44 \cdot 10^{8}$ & $\sim 1.44 \cdot 10^{8}$ & $\sim 1.44 \cdot 10^{8}$ & $\mathrm{~N} / \mathrm{A}$ \\
\hline
\end{tabular}

Even after the components are minimized, the resulting system still has over $3.4 \cdot 10^{37}$ states.

\section{Modeling Effort}

The tables in the previous section convey information about the computational effort needed to minimize the models we developed. However, the effort expended in a verification project is not only due to the time elapsed between "pushing the button" and "getting a result", but also the manpower needed to construct the models in the first place. The work involved in model creation is an iterative process involving inspecting the requirements and simulating and refining the model under development. It should also be noted that requirements expressed in a natural language like English are imprecise, and often reasonable assumptions have to be made with respect to the constructed models. And Although this modelconstruction phase is laborious and frequently frustrating, its benefit cannot be overemphasized. The exercise of formally encoding a system brings to the fore many ambiguities that otherwise would slip into the system design; this process of model elicitation, if fed back to the requirements team, can typically also lead to better and more precise encodings of requirements. 
Based on the above observations, evaluating a methodology requires an account the human effort needed to construct the models. In the case of this project, it took an approximate of 60 man-hours to settle on a reference model of the CARA system and about ten man-hours to encode it in the CWBNC. However, the reference-model creation and the encoding/validation of the model went on side-by-side and involved many iterations. It should also be noted that the effort would have been significantly less if we had been able to interact more with the actual system designers in order to clarify ambiguities in the system requirements.

\section{Verifying CARA: The Unit-Based Approach}

The previous section gave a sense of our Temporal CCS model of CARA. In this section we describe our efforts to check specific properties of the model. These properties were extracted from the CARA requirements documents given to us by WRAIR researchers [3-5].

Our initial intention was to take the model of Section 5, translate requirements into the modal mu-calculus [21], and use the CWB-NC's model checker to check which properties held and which did not. This approach proved untenable, owing to the large size of the model, even after the individual components were minimized.

Instead, we pursued a strategy we refer to as unit verification, and which was also used in [15], although it was not referred to by this name in that paper. Such an approach is feasible when requirements are given as scenarios ("whenever a certain behavior is observed, take these actions") that involve small subsets of the over-all components in the system. The essential idea is to encode the relevant scenario as a process that interacts with the components in question and then check whether the outcome of the scenario is "successful" or not.

In the rest of this section we first define unit verification more precisely and talk about the properties that can be checked using it. We then report on our experiences using unit verification to study the CARA model.

\subsection{Unit Verification}

Unit verification derives its name from unit testing. In unit testing, software modules are first tested in isolation before being assembled into full systems. In order to test a module that may, in the final system, not have an interface to the external environment, one typically constructs a test harness that drives the execution of the software under test. Unit testing is frequently used in software projects because it gives engineers an ability to detect bugs at the module level, when they are easier to diagnose and fix. For unit testing to work, of course, one must have module-level requirements at hand so that test results can be analyzed.

In unit verification, the set-up is very similar to unit testing: single modules are verified in isolation using "harnesses" to provide the stimuli that the other modules in the system (or the external environment) would generate once the module is deployed. As with unit testing, this approach requires the presence module-level requirements so that results can be correctly interpreted.

\subsubsection{Trace Properties}

Unit verification deals primarily with trace properties: properties of system executions. In this section we sketch a basic theory of such properties.

As executions may be thought of as sequences, we use standard mathematical operations on sequences in what follows: if $A$ is a set, then $A^{*}$ is the set of sequences whose elements come from $A$, if $\sigma, \sigma^{\prime}$ are sequences then $\sigma \cdot \sigma^{\prime}$ is the sequence obtained by concatenating them in the given order, $\epsilon$ is the empty sequence, etc.

Definition 5. Let $M=\left\langle S, A, \longrightarrow, s_{I}\right\rangle$ be a DTLTS.

1. Let $s, s^{\prime} \in S$ be states and $\sigma \in\left(A_{\{1\}}\right)^{*}$ be a sequence of (non- $\tau$ ) transition labels. Then

$$
s \stackrel{\sigma}{\Longrightarrow} s^{\prime} \text { if }\left\{\begin{array}{l}
\sigma=\epsilon \text { and } s \stackrel{\epsilon}{\Longrightarrow} s^{\prime} \text { in Def. 3(1), or } \\
\sigma=a \cdot \sigma^{\prime} \text { and } \exists s^{\prime \prime} \in S . s \stackrel{a}{\Longrightarrow} s^{\prime \prime} \stackrel{\sigma^{\prime}}{\Longrightarrow} s^{\prime} .
\end{array}\right.
$$

2. The language, $L(M, s)$, of $s \in S$ is defined by:

$$
L(M, s)=\left\{\sigma \in\left(A_{\{1\}}\right)^{*} \mid s \stackrel{\sigma}{\Longrightarrow} s^{\prime} \text { some } s^{\prime} \in S\right\} .
$$

3. The language, $L(M)$ of $M$ is defined by:

$$
L(M)=L\left(M, s_{I}\right)
$$

The language of a state in a DTLTS contains the sequences of visible actions / clock ticks that a user can observe as execution of the DTLTS proceeds from the state. The language of the DTLTS is just the language of the start state.

In this case study the properties we are concerned with involve system executions and come in two varieties: safety and quasi-liveness. These are defined as follows.

Definition 6. Let $M=\left\langle S, A, \longrightarrow, s_{I}\right\}$ be a DTLTS.

1. A safety or quasi-liveness property over $A$ is any subset of $\left(A_{\{1\}}\right)^{*}$.

2. $M$ satisfies safety property $\mathcal{S}$ if and only if $L(M) \subseteq \mathcal{S}$.

3. $M$ satisfies quasi-liveness property $\mathcal{Q}$ iff for every $\sigma, s$ such that $s_{I} \stackrel{\sigma}{\Longrightarrow} s$, there exists $\sigma^{\prime} \in L(M, s)$ such that $\sigma \cdot \sigma^{\prime} \in \mathcal{Q}$.

Intuitively, a safety property contains "allowed" execution sequences; a system satisfies such a property if all the system's executions are allowed. A quasi-liveness property is more complicated: it contains sequences that a system, regardless of the current execution it has just performed, should be able to "complete". We call these properties quasi-liveness because the definition of satisfaction does not require that such "complete-able" executions actually be completed, only that the system always be capable of doing so. At first blush, 
this requirement may not seem strong enough to ensure "liveness" in the tradition sense. However, our intuition is that, if a quasi-liveness property is satisfied by a system, then in any "reasonable" run-time setting employing some kind of fair scheduling, a "complete-able" execution will eventually be completed. These definitions are inspired by, but differ in several respect from, the classic definitions of safety and liveness in [6].

\subsubsection{Defining Unit Verification}

The unit verification approach we advocate in this paper may be used to check whether a system satisfies safety / quasiliveness properties as defined in the previous section. The method consists of the following general steps, where $M$ is the module being analyzed and $P$ is the property.

1. Construct a verification harness $H_{P}[]$.

2. Plug $M$ into $H_{P}[]$, yielding a new system $H_{P}[M]$.

3. Apply a check to $H_{P}[M]$ to see if $M$ satisfies $P$ or not.

The checks applied to $H_{P}[M]$ depend on whether $P$ is a safety or quasi-liveness property.

In the remainder of this section we flesh out the unit verification approach in the context of Temporal CCS. We define what verification harnesses $H_{P}$ [] are and the checks that are applied on $H_{P}[M]$. We also discuss optimizations to the procedure that can be undertaken to improve (often greatly) performance.

Verification Harnesses in Temporal CCS. Verification harnesses are intended to "focus attention" on interesting execution paths in a module being verified. The general form of a verification harness is:

\section{$\left(V_{P} \mid[]\right) \backslash \Lambda$}

where $\Lambda$ is the set of all communication labels, $V_{P}$ is a (deterministic) Temporal CCS expression that we sometimes call a verification process, and [] is the "hole" into which the module to be verified is to be "plugged".

As a practical matter, in our CARA work we did not derive verification processes from properties; instead, based on our reading of system requirements we directly constructed the $V_{P}$ components of our test harnesses and used them as our representations of properties. We therefore explain how properties may be extracted from DTLTSs in what follows.

In our setting, verification processes draw their visible actions from $A^{\text {TCCS }}$ (the Temporal CCS action set introduced in Section 3.1) augmented with two special actions, good and bad. The latter are used to determine what properties a verification process defines. Recalling that the semantics of Temporal CCS specifies how Temporal CCS expressions may be "compiled" into single DTLTSs, in what follows we assume that our verification processes are single DTLTSs.

In order to characterize the properties associated with a verification process $V$, we first note that $V$ is intended to run in parallel with the module being verified. In order to guide the behavior of the module, $V$ must synchronize with the modules actions, meaning that when $V$ wants the module to perform an input action $a, V$ must perform the corresponding output $\bar{a}$. In general, then, since module properties refer to the actions in the module, to associate a module property with $V$ we need to reverse input / output roles in $V$ 's execution sequences. To make this precise we introduce the following notation.

Definition 7. Let $\sigma \in\left(A_{\{1\}}^{\mathrm{TCCS}}\right)^{*}$ be a sequence of externally controllable actions. Then $\bar{\sigma} \in\left(A_{\{1\}}^{\text {TCCS }}\right)^{*}$ is defined inductively as follows, where $a \in A_{\{1\}}^{\mathrm{TCCS}}$.

1. $\bar{\epsilon}=\epsilon$

2. $\overline{a \cdot \sigma^{\prime}}=\bar{a} \cdot \overline{\sigma^{\prime}}$, where $\overline{\bar{\lambda}}=\lambda$ and $\overline{1}=1$.

A verification process $V$ defines both a safety property, $\mathcal{S}(V)$, and a quasi-liveness property, $\mathcal{Q}(V)$, as follows.

$$
\begin{aligned}
\mathcal{S}(V)= & \left\{\sigma \in\left(A_{\{1\}}\right)^{*} \mid\right. \\
& \left.\nexists \sigma_{1}, \sigma_{2} \cdot \bar{\sigma}=\sigma_{1} \cdot \sigma_{2} \wedge \sigma_{1} \cdot \text { bad } \in L(V)\right\} \\
\mathcal{Q}(V)= & \{\bar{\sigma} \mid \sigma \in L(V) \wedge \sigma \cdot \operatorname{good} \in L(V)\}
\end{aligned}
$$

Intuitively, if bad is possible as the next action in an execution then the execution, and all possible ways of extending it, are removed from $\mathcal{S}(V)$. Similarly, action sequences leading to the enabling of good are included in the property $\mathcal{S}(V)$.

Defining Safety and Quasi-Liveness Checks. From the structure of $H_{P}[]$ one can see that the only actions that $H_{P}[M]$ can perform for any $M$ are $\tau, 1$, good and bad. This is due to the fact that $H_{P}[M]=\left(V_{P} \mid M\right) \backslash \Lambda$, and the $\backslash \Lambda$ operator prevents all but these actions from being performed. This fact greatly simplifies the task of checking whether or not a safety / quasi-liveness property encoded within a verification process holds of a module.

Theorem 1. Let $M$ be a Temporal CCS system model and $V$ be a verification process. Then the following hold.

1. $M$ satisfies $\mathcal{S}(V)$ if and only if $(V \mid M) \backslash \Lambda \models[\text { bad }]_{\{\tau, 1\}}$ ff

2. $M$ satisfies $\mathcal{Q}(V)$ if and only if $(V \mid M) \backslash \Lambda \models[\epsilon]_{\{\tau, 1\}}\langle\text { good }\rangle_{\{\tau, 1\}} t t$

Proof. Follows immediately from the definitions of $\mid, \backslash L, \mathcal{S}$ and $\mathcal{Q}$. The determinacy of $V$ is important.

This theorem says that the correct "check" for the safety property encoded in a verification process $V$ is to see whether or not the "plugged-in" verification harness, $(V \mid M) \backslash \Lambda$, forever disables the bad action: formula [bad $]_{\{\tau, 1\}}$ ff holds exactly when there are no execution sequences consisting of $\tau$ 's, 1 's and a single bad action. Likewise, to check if $V$ 's liveness property holds of $M$, it suffices to check that $(V \mid M) \backslash \Lambda$ satisfies $[\epsilon]_{\{\tau, 1\}}\langle\text { good }\rangle_{\{\tau, 1\}} \mathrm{tt}$ : if so, then regardless of what $M$ does, there is still a possibility of $(V \mid M) \backslash \Lambda$ evolving to a state in which good is enabled.

In some cases, it may be more natural to "look for bugs" rather than to try to prove the nonexistence of bugs. This might be the case if, for example, one strongly suspects erroneous behavior. To determine if a module violates a verification process's safety property, one may perform the following check:

$$
(V \mid M) \backslash \Lambda \models\langle\text { bad }\rangle_{\{\tau, 1\}} \mathrm{tt}
$$


If the answer is "yes" then a violation exists. Similarly, one may check

$$
(V \mid M) \backslash \Lambda \models\langle\epsilon\rangle_{\{\tau, 1\}}[\mathrm{good}]_{\{\tau, 1\}} \mathrm{ff}
$$

to test whether or not $M$ violates $V$ 's quasi-liveness property.

Optimizations. So far our basic unit verification methodology consists of the following steps.

1. Formulate a verification process $V$.

2. To check whether or not $V$ 's safety / quasi-liveness property holds of $M$, check whether or not simple modal mucalculus formulas hold of $V$ "running in parallel with" $M$.

In our case study work, we found that two simple optimizations greatly facilitated this process; we describe these here.

Minimization. Checking whether or not a mu-calculus property holds of a system requires, in general, a search of the system's state space. Reducing the size of this state space thus reduces the time required by this search. In the case of $(V \mid M) \backslash \Lambda$, one way to reduce states in the parallel composition is to reduce states in $V$ and $M$ by minimizing them with respect to observational equivalence.

Action Hiding. In general, the properties we confronted in the CARA study only focused on a few actions in the module being tested. For example, in a property of the form "whenever a blood-pressure source fails, an alarm should be sounded", actions not related to detecting failure and raising an alarm are unimportant. Mathematically, this is reflected in the structure of a verification process: every state has a selfloop for every unimportant action, since such actions do not "affect" the verification result.

This observation can be exploited to reduce the state space of $(V \mid M) \backslash \Lambda$ even further as follows.

1. Partition $\Lambda$ into a set $I$ of "interesting" labels and a set $U=\Lambda-I$ of "uninteresting labels."

2. Hide actions involving uninteresting labels in $M$, creating $M^{\prime}=M\lfloor U\rfloor$ (and likewise for $V$, creating $V^{\prime}$ ).

3. Minimize $M^{\prime}$ and $V^{\prime}$ and perform the safety / quasi-liveness check on $\left(V^{\prime} \mid M^{\prime}\right) \backslash I$.

Hiding actions turns them into $\tau$ 's; this process enhances possibilities for minimization, since observational equivalence is largely sensitive only to "visible" computation.

In the CARA study, we usually constructed $V^{\prime}$ directly, without minimizing; so the benefits of this optimization accrue mostly in the construction of $M^{\prime}$.

A note of caution is in order here. Hiding actions in Temporal CCS turns them into $\tau$ actions. Since Temporal CCS has the maximal progress property (cf. Def. 2 in Section 3), introducing cycles of $\tau$ 's via hiding can cause timing behavior to be suppressed (a $\tau$-cycle can cause "time to stop"). When hiding actions, care must be taken not introduce such loops, or divergences, as they are often called. The CWB-NC model checker may be used to check for the presence or absence of divergences.
Putting It All Together. What follows summarizes our general approach to unit verification. To check a safety or quasiliveness property of a module $M$ :

1. Formulate an appropriate verification process $V$.

2. Identify the interesting $(I)$ and uninteresting $(U)$ labels in $V$.

3. Form $M^{\prime}=M\lfloor U\rfloor$, which hides the actions involving uninteresting labels in $M$. Make sure no divergent behavior is introduced into $M^{\prime}$.

4. Minimize $M^{\prime}$, yielding $M^{\prime \prime}$.

5. Do the same on $V$ if necessary, yielding $V^{\prime \prime}$.

6. To check $V$ 's safety property: determine whether or not $\left(V^{\prime \prime} \mid M^{\prime \prime}\right) \backslash I \models[\mathrm{bad}]_{\{\tau, 1\}} \mathrm{ff}$.

7. To check $V^{\prime}$ 's quasi-liveness property: determine whether or not $\left(V^{\prime \prime} \mid M^{\prime \prime}\right) \backslash I \models[\epsilon]_{\{\tau, 1\}}\langle\text { good }\rangle_{\{\tau, 1\}}$ tt.

\subsubsection{Tool Support.}

The CWB-NC tool includes several routines that support the unit verification procedure described above. Primary among these are two different routines for checking whether or not mu-calculus formulas hold of systems. One, the basic model checker, returns a "yes / no" answer quickly. Another, the search utility, searches from the start state of a system for another state satisfying a given property: if one is found, then the simulator is "loaded" with a shortest-possible sequence of execution steps leading from the start state to the state in question. This enables the user to step through the given execution sequence to examine how the found state was reached. The search utility is especially useful in the "bug searching" procedure mentioned earlier. In particular, to determine if a module $M$ violates the safety property of verification process $V$, it suffices to search from the start state of $(V \mid M) \backslash \Lambda$ for a state satisfying $\langle\mathrm{bad}\rangle \mathrm{tt}$ (a mu-calculus formula holding of states from which bad is immediately enabled). If such a state is found, then the safety property is violated, and the execution sequence loaded into the simulator may be examined to determine why. In the case of quasi-liveness, the same process may be searched for a state satisfying [good $]_{\{\tau, 1\}} \mathrm{ff:}$ if such a state exists then the quasi-liveness property is violated.

The tool also contains a sort utility that, given a Temporal CCS system description, returns all the externally controllable (i.e. non- $\tau$ ) actions the system can performed. The sort command provides a convenient utility for checking whether or not a safety property holds: check whether or not the harnessed process's sort contains bad. It also may be used to check for violations of quasi-liveness properties: if the harnessed process's sort does not contain good, then the property is violated. The latter is only a sufficient condition: just because good is in the sort of such a process does not guarantee that the quasi-liveness property is satisfied.

The CWB-NC also includes a routine for minimizing systems with respect to observational equivalence. 
Table 3. Properties Checked on CARA Using Unit Verification.

\begin{tabular}{|c|c|c|}
\hline Number & Type & Property \\
\hline 1 & Safety & "Two successive Emf checks occur no more than five seconds apart." \\
\hline 2 & Quasi-liveness & $\begin{array}{l}\text { "If an override question is asked and then not answered, a corroboration cycle will never start } \\
\text { again." }\end{array}$ \\
\hline 3 & Safety & $\begin{array}{l}\text { "The alarm module reacts properly to errors, i.e. a high-alarm condition results in a high ring and a } \\
\text { low-alarm condition results in a low ring." }\end{array}$ \\
\hline 4 & Safety & "When an override question is pending, the system cannot take a new input parameter." \\
\hline 5 & Safety & "When an alarm condition is present, the system cannot move from manual to auto-control mode." \\
\hline 6 & Safety & "When the system is in an error state, no new input parameter will be accepted." \\
\hline
\end{tabular}

\subsection{Analyzing CARA Using Unit Verification}

In this section, we concentrate on half a dozen properties of CARA that we investigated using unit verification.

Table 3 summarizes the properties discussed in more detail below. The properties were all derived from the CARA requirements documents [3-5]. In each case, the property focuses on the localized behavior of one, and in one case two, modules.

Table 4 summarizes the results obtained using unit verification. The data reported includes the property, the size of the relevant "harnessed module", the CWB-NC command used to check the relevant safety / quasi-liveness property ("chk" for model checking, "search" for the state-space searching procedure described above), the outcome of the check, and the seconds of CPU time needed. The workstation used to conduct the experiments is the same as the one mentioned in Section 5.

As can be seen from the reported results, Properties 1 and 2 fail to hold of the model; we view these as products of inconsistencies in the requirements. In the rest of this section, we explain the sources of these anomalies and describe in more detail the verification processes used to uncover the problems. We also give more detail on the other properties.

\subsection{Property 1: Amok Time}

To explain the source of the problem with Property 1, we mention some design requirements from [5].

1. Impedance and back Emf values are polled values. (vide Q66)

2. When a polling request fails, retry two more times at onesecond intervals. Only if three attempts fail should an alarm be raised. (vide Q74)

3. The following sequence of events must occur at five-second intervals. (vide Q70)

(a) Check Emf

(b) Update display of flow rate

(c) Check impedance value

We claim that these three requirements are not compatible: if Requirements 1 and 2 are satisfied, then there is a case when Requirement 3 will be violated.

To justify our claim, we first give an informal argument as to why there would be a violation. Then we formally prove it by constructing a suitable verification process that, when combined with the appropriate module, is capable of emitting a bad action.

Let us consider the following scenario. An Emf check starts. The first reading at the end of one second (since Emf is a polled signal) is an error. By Requirement 2 it is checked again and again gets an error. (So far two seconds of time have elapsed.) Then on the third attempt, a valid Emf reading is obtained (time elapsed: three seconds). Then, based on Requirement $3 \mathrm{~b}$ the flow rate is adjusted. Since no data for updating flow rates was given, we assume it is instantaneous. Then the impedance check is performed. Since that too is a polled value like the Emf, it follows the same discipline of three bad readings before an error is flagged. Like the Emf, let the first two readings, at a one-second intervals each, give errors and the third reading give a good value. So the time elapsed is six seconds. So even if an Emf check starts at that second, six seconds have elapsed since the initiation of the last Emf check. This violates Requirement 3, which states that at most five seconds can have elapsed. The sequence of events described is valid, and a sequence in which an alarm is not raised. So it cannot be justified as an error run which could be assumed to violate some other constraints. What we have is a valid run violating the constraints imposed by the specifications by making the time elapsed between two successive Emf checks to be six seconds.

To show this formally, we apply unit verification to the relevant module, which in this case is EmfChecker. The transitions we are concerned with relate to those involving erroneous (Emf_o) and valid (Emf_1) Emf readings and erroneous (Imp_0) and valid (Imp_1) impedance readings. All other transitions in EmfChecker are hidden, i.e. converted into $\tau$-transitions.

The verification process itself is a Temporal CCS process constructed using the two DTLTSs given in Fig. 5. DTLTS Timer awaits the enabling of its start action (idling loops are omitted) and then counts down five seconds. At any time during this five seconds, if it is capable of performing its end action, then the timer is reset. If time expires and end happens, then bad is performed. This timer captures the fivesecond upper bound in Requirement 3.

DTLTS Test, on the other hand, starts the timer by performing $\overline{\text { start }}$ and then engages in the sequence of actions described above: two successive erroneous reading of the Emf 
Table 4. Results of Properties Checked on CARA.

\begin{tabular}{|l|r|r|l|l|l|}
\hline Property & \multicolumn{2}{|c|}{ Harness size } & CWB-NC & & $\begin{array}{l}\text { Time } \\
\text { Number }\end{array}$ \\
\cline { 2 - 3 } & States & Transitions & Command & Result & (sec.) \\
\hline 1 & 101 & 127 & search & False & 0.280 \\
2 & 5,423 & 7,247 & chk & False & 17.820 \\
3 & 65 & 107 & chk & True & 0.190 \\
4 & 32 & 72 & chk & True & 0.210 \\
5 & 85 & 757 & chk & True & 3.440 \\
6 & 18 & 32 & chk & True & 0.070 \\
\hline
\end{tabular}
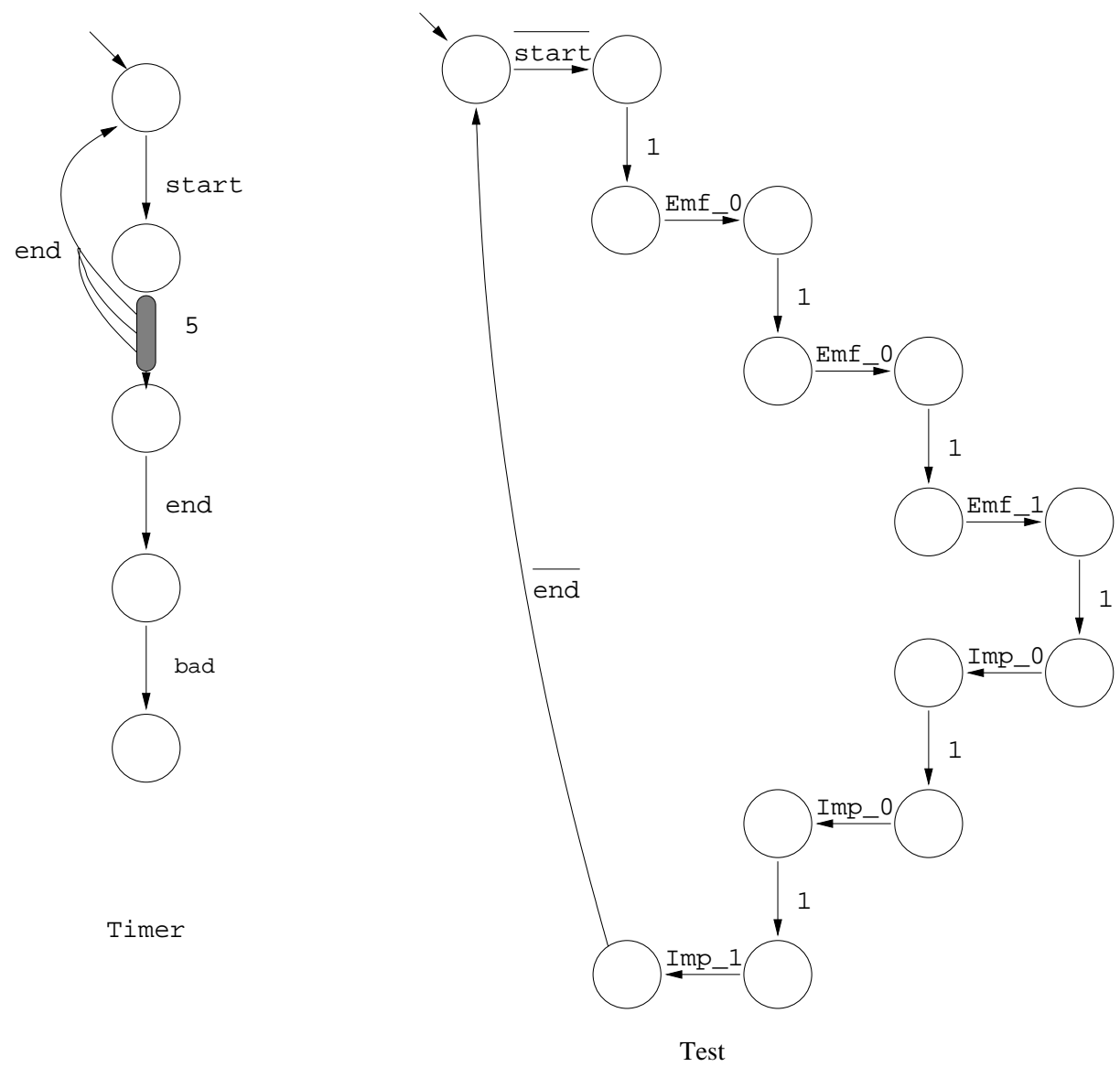

Fig. 5. DTLTSs Timer and Test Used in Verification Process for Property 1.

followed by a valid one, and likewise for the impedance. At the end, it stops the timer by emitting $\overline{\text { end. }}$

The whole verification process is then given by the Temporal CCS expression

$$
\text { (Timer } \mid \text { Test }) \backslash\{\text { start, end }\} \text {. }
$$

The restriction operator ensures that only Test can start and stop Timer. The net effect of this process is to attempt to perform a valid six-second execution sequence on EmfChecker.

The results in Table 4 vindicates our intuitions: Requirement 3 is violated.

As a final observation, we note that this use of unit verification may be seen as a formalized counterpart to debugging.
In this case we informally observed what appeared to be a problem and then constructed a verification harness that exposed it.

\subsection{Property 2: Locked in Life}

As in the previous property, we first give an intuitive formulation of the problem. The first relevant requirement for this property is given as Q118 in [5], where the following question is asked and answered.

What should be done if the 30-minute timer activities are pending due to an unanswered override question, 
and another 30-minute timer expires ?

The system should continue waiting.

The second relevant requirement is mentioned in Q109 in the same document:

What should be done to current corroboration attempts if another higher priority source starts reporting?

The current corroboration attempts must complete before the new sources will be corroborated. This means that the override question must be answered before corroboration is attempted for any new source.

The problem with this is immediately clear: what would be the situation if the override question is never answered? By the above requirement, the system should continue waiting for successive 30 -minute intervals forever, meaning that the system is in a live-lock. Even when a source comes up, the system ignores it and keeps waiting (vide Q109). The implications are severe. For example, suppose that the cuff pressure goes down, the override question is asked, and it is not answered. When the cuff comes up again, the corroboration question is no longer relevant (as the original corroboration question was initiated by the cuff becoming invalid). But the question, despite being irrelevant, is still being asked. On top of that, the system does not take any action based on the fact that the cuff has come up and stops corroboration until the irrelevant override question is answered. Since the purpose of this system is to operate with minimal manual intervention, it seems a reasonable assumption that there might be scenarios (e.g. a single care-giver attending to a large number of wounded soldiers) when a particular override question may remain unanswered for significant periods of time. For that entire duration, all corroboration efforts will stop and the system will take no steps to resolve the override question. Even if the source comes up, the system will not be receptive to it. Thus the pressure-control subsystem would stop working until someone answers the override question.

To establish that this live-lock can indeed occur, we focus on the Corrobcontrol module of Table 1, which handles corroboration issues. The associated verification process is given as a single DTLTS in Fig. 6, which "asks" the override question, awaits a blood-pressure reset action, and then performs the good action. The reset action is never enabled, however, by the corresponding action in Corrobcontrol, and thus no good action is every performed by the harnessed process. Again, our intuitions are confirmed: the requirements contain an inconsistency.

A simple solution for this can be given. There should be a default answer to the override question which can be changed at any time by the care-giver. If an override question is asked, the system would wait for a specific time. If no resolution of the override question is made during that time by care-giver input, the override question would "time out" and the default answer to the override question would be assumed. The system then can proceed and not be live-locked any longer.

\subsection{Properties 3-6}

In contrast with Properties 1 and 2, Properties 3-6 hold of the relevant "modules" of the system. In the case of Property 3, the module to which the property is applicable is Alarm (cf. Table 1). The verification process is depicted in Fig. 7 (idling loops are omitted). Using the CWB-NC model checker, one can determine that $\mathrm{Al}$ a rm responds correctly to alarm-raising stimuli.

Property 5 is of interest because the "unit" to which unit verification is being applied consists of two modules from Table 1: Display and ModeControl. This is because the requirement from which the property is drawn refers to actions in Display (alarms) and ModeControl (mode switches). The unit to which the relevant verification process is applied has form

$$
\text { Display | ModeControl. }
$$

This example illustrates another feature of unit verification, namely, that "units" may consist of several individual "modules".

\section{Discussion and Related Work}

As should no doubt be evident by now, CARA is a non-trivial system of significant complexity. Needless to say, modeling it posed many challenges. One of the main problems lay in the requirements themselves. Having been written over a period of time, several inconsistencies had crept in, despite the best efforts of the WRAIR researchers to apply rigorous, cleanroombased techniques to requirement capture [23]. A more precise design language that would overcome the natural ambiguities and unstructuredness of a textual description of such a complex system would have been very helpful in this regard. Another problem was that medical terms in the specification document were not defined. This made it problematic for people who were not domain experts in the field of medical instrumentation to understand the documents.

An important consideration was the tool to be used in the analysis. Since the system's operation was heavily dependent on time and the most important properties were temporal in nature, the modeling language had to be rich enough to support time in an elegant and simple way. Another requirement was that the language should support a hierarchical architecture. Hence the modeling language used was Temporal Calculus of Communicating Systems, a timed extension of the CCS language that contains support for concurrent hierarchical state machines and discrete time. As this language is implemented as a front-end for the Concurrency Workbench of the New Century, the natural choice for the tool was the CWB-NC.

Once the obvious ambiguities in the requirements documents were resolved, the immediate problem lay in finding suitable abstractions so that the model's representation would be amenable to model checking. If the system were modeled in full detail, the state space became so large (due to state 


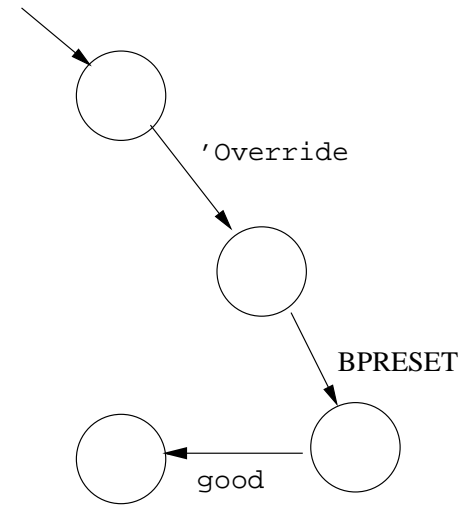

Fig. 6. Verification Process for Property 2.

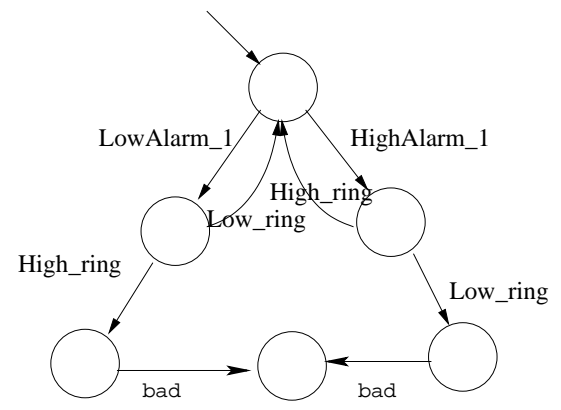

Fig. 7. Verification Process for Property 3.

explosion) that the system could not be analyzed in the tool being used. The first attempts to reduce the system state space were the application of the standard techniques of abstraction and simplification.

In the process of trying to construct formal models of the CARA system in the rather low-level languages provided by formal verification tools like the CWB-NC, the abstractions and simplifications that were done made it very difficult to make a strong case that the formalizations faithfully captured "the CARA system," or that properties formally verified about the model had any implications for the real system. Keeping in mind this, it was decided to keep abstractions and simplifications to a minimum and not compromise on details. Consequently, the modeling became intricate and sufficiently detailed so that the system could be captured in its entirety to remove doubts that the modeled system was actually the CARA system in question.

Another possible source for a state explosion lay in the representation of time. Each clock tick was represented by a state. So a delay of 15 time units would mean 15 states. And since there are many such clocks working in parallel, the state space became enormous. An option would have been to model time with non-determinism. But then again it would defeat the utility of analysis as the interesting properties were temporal in nature.
The challenges posed by all these design decisions led us to investigate novel ways to reduce the state-space of the models constructed. The solution was unit verification, which constructs only relevant parts of the state space. When the principle of unit verification was applied to the CARA system, the results were spectacular. The hitherto intractable modelchecking efforts became very simple on the individual components. Results were obtained in real-time. The CWB-NC has automated ways for finding the externally visible actions of a component. So the modeling effort involved hiding the transitions not relevant to the property. This could be done in a automated way using the workbench. Then a suitable test had to be crafted, and the model checker used to find out if the test ended in a success or failure test.

Overall fifteen properties were verified. Most of these properties were taken from the requirements documents and were sanity checks. By sanity check, we mean properties which relate to proper working of alarms, notification of pressure losses to the appropriate modules and timings of corroboration efforts. Other properties related to switching between manual and auto-control stages. Some of these properties could be proved without unit verification on the modules but even in those cases the state space lay in ten thousands. Other modules could just not be handled in a reasonable time. But once unit verification was applied state spaces shrank to the order 
of hundreds with the result coming in seconds as compared to minutes, hours or sometimes not at all!

A natural question that arises concerns the difficulty of constructing verification processes from properties. In our work we tended to construct the verification processes directly; the "properties" we were encoded were not formalized independently of the processes. This approach turned out to be very natural for us: rather than devising properties declaratively and then "operationalizing" them as verification processes, we coded the operationalizations directly. Another benefit of this approach is that the "property language" and the "modeling language" are the same, meaning that a user need only master one notation rather than two.

\section{Related Work}

The CARA system developed by WRAIR has been extensively studied by research groups at the State University of New York at Stony Brook (USB), Stanford University, the University of Pennsylvania, North Carolina State University and the New Jersey Institute of Technology. At USB in particular, several different threads of work of emerged, and we comment on these here.

Arne Skou of Aalborg University in Denmark, in conjunction with one of the authors of this paper (Arnab Ray), developed some rudimentary models of the CARA system during the Spring of 2001, when Skou was visiting USB [31]. He used the UPPAAL [20] tool to model and verify some of the simplified models. UPPAAL provided a much more visually pleasing and intuitive interface to the user than CWBNC. But it was felt that its analytical power was weaker, owing to its modeling of continuous, rather than discrete, real time: in particular, minimization of system descriptions was difficult to undertake. For the CARA case study the CWB-NC proved more versatile than UPPAAL; however, the tool suffers from a text-based interface in the sense that simulating the system is not a visually attractive experience. But what it lacks in an intuitive GUI it makes up for in its expressive power and analysis muscle. And the rich GUI of UPPAAL was not always a blessing. It was observed that while designing components with many states and inputs, the graphical input language of UPPAAL became very difficult to work with because it became hard to maintain the global overview of the component. It was precisely due to this reason that a detailed design of the blood-pressure control unit could not be given in UPPAAL.

Another parallel effort in analyzing the CARA system was undertaken by Gene Stark at USB. His approach was to create a JAVA applet which simulated the low level functioning of the CARA system. The applet was based on a formal CARA model which he constructed for the purpose, and it provided a "control-panel" approach to simulating CARA, in which users could "press buttons" and otherwise undertake activities defined in the CARA documents. However, this model, while precise, is not "formal" in the traditional sense of the word, and no verification tools exist that would permit e.g. model checking to be applied to it.
Arnab Ray was also associated with the efforts of the Stanford group when he was a summer intern there in the Summer of 2001. There the work of modeling was done using the Stanford Temporal Logic Prover [25], which threw up new challenges in the modeling effort since STEP was a infinite-state deductive system where properties were proved by theorem proving and not by model checking.

Other researchers have also studied techniques similar to unit verification for checking properties of systems. Elseaidy, Cleaveland and Baugh [15] explore a method based on $o b$ server processes for checking safety properties of real-time system; the approach is essentially that of safety-property checking described in this paper, although no mention is made of liveness there. In a series of papers, most notably $[9,32]$, Cheung, Giannapolou and Kramer describe the use of "property automata", which run in parallel with a system to be verified, and give algorithms for determining whether safety and liveness properties hold in this setting. No mention is made of real time in that work, however. Finally, work on automaton-based model checking [22] is also related. In this approach formulas to be checked are converted into automata that "monitor" the states a system enters in order to determine whether properties are violated or not. That work, however, focuses on unlabeled transition-system models of systems, in contrast with the work in this paper.

\section{Conclusions and Directions for Future Research}

In this paper we have focused on modeling and analyzing properties of the Computer-Aided Resuscitation Algorithm (CARA), an automated cardio-pulmonary resuscitation device intended for deployment in battlefield situations. We developed a detailed model in the Temporal CCS modeling language as supported by the Concurrency Workbench of the New Century, a verification tool. The model proved too large to analyze in toto; we consequently focused on applying a technique, unit verification, that permits collections of system components to be analyzed independently of the rest of the system. When these collections are small, unit verification offers an attractive alternative to traditional global model checking. This observation was borne out in the CARA case study, in which checks of individual "unit" properties typically took only fractions of a second to perform.

Unit verification is not a panacea for automated verification: it is likely to be of most use when there are detailed, module-level requirements for the system, as there was with CARA. As the number of modules that must be considered for a property increases, the utility of unit verification vis à vis traditional model checking is likely to wane.

As future work, it would be interesting to explore more carefully what kinds of properties can be checked using unit verification. Another case study would also be useful as a means of further exploring the utility of the technique. 


\section{References}

1. www. lstat.com.

2. http://www.infusiondynamics.com/rpump.html.

3. Hazards analysis. 1999.

4. CARA increment 3 formal numbered requirements. 2001.

5. CARA pump control software-question / answer document. 2001.

6. B. Alpern and F. Schneider. Recognizing safety and liveness. Distributed Computing, 2(3):117-126, 1987.

7. J.C.M. Baeten and W.P. Weijland. Process Algebra, volume 18 of Cambridge Tracts in Theoretical Computer Science. Cambridge University Press, Cambridge, England, 1990.

8. T. Bolognesi and E. Brinksma. Introduction to the ISO specification language LOTOS. Computer Networks and ISDN Systems, 14:25-59, 1987.

9. S. C. Cheung, D. Giannakopoulou, and J. Kramer. Verification of liveness properties using compositional reachability analysis. In Proceedings of ESEC/FSE, Zurich, Switzerland, 1997.

10. E.M. Clarke, O. Grumberg, and D.A. Peled. Model Checking. MIT Press, Cambridge, Massachusetts, 2000.

11. R. Cleaveland, E. Madelaine, and S. Sims. A front-end generator for verification tools. In E. Brinksma, R. Cleaveland, K.G. Larsen, and B. Steffen, editors, Tools and Algorithms for the Construction and Analysis of Systems (TACAS '95), volume 1019 of Lecture Notes in Computer Science, pages 153-173, Aarhus, Denmark, May 1995. Springer-Verlag.

12. R. Cleaveland, J. Parrow, and B. Steffen. The Concurrency Workbench: A semantics-based tool for the verification of finite-state systems. ACM Transactions on Programming Languages and Systems, 15(1):36-72, January 1993.

13. R. Cleaveland and S. Sims. The NCSU Concurrency Workbench. In R. Alur and T. Henzinger, editors, Computer Aided Verification (CAV '96), volume 1102 of Lecture Notes in Computer Science, pages 394-397, New Brunswick, New Jersey, July 1996. Springer-Verlag.

14. R. Cleaveland and S. Sims. Generic tools for verifying concurrent systems. Science of Computer Programming, 42(1):39-47, January 2002.

15. W. Elseaidy, R. Cleaveland, and J.W. Baugh Jr. Modeling and verifying active structural control systems. Science of Computer Programming, 29(1-2):99-122, July 1997.

16. J.-C. Fernandez. An implementation of an efficient algorithm for bisimulation equivalence. Science of Computer Programming, 13:219-236, 1989/1990.

17. M.C.B. Hennessy. Algebraic Theory of Processes. MIT Press, Boston, 1988.

18. C.A.R. Hoare. Communicating Sequential Processes. PrenticeHall, London, 1985.

19. P. Kanellakis and S.A. Smolka. CCS expressions, finite state processes, and three problems of equivalence. Information and Computation, 86(1):43-68, May 1990.

20. Paul Pettersson Kim G. Larsen and Wang Yi. Uppaal in a nutshell. Springer International Journal of Software Tools for Technology Transfer 1(1+2), 1997.

21. D. Kozen. Results on the propositional $\mu$-calculus. Theoretical Computer Science, 27(3):333-354, December 1983.

22. O. Kupferman, M.Y. Vardi, and P. Wolper. An automatatheoretic approach to branching-time model checking. Journal of the Association for Computing Machinery, 47(2):312-360, March 2000.
23. R. Linger. Cleanroom process model. IEEE Software, 11(2):50-58, March/April 1994.

24. Z. Manna and A. Pnueli. The Temporal Logic of Reactive and Concurrent Systems. Springer-Verlag, Berlin, 1992.

25. Zohar Manna and the STEP team. Step: The stanford temporal prover (educational release), user's manual. Technical report STAN-CS-TR-95-1562, 1995.

26. R. Milner. Communication and Concurrency. Prentice-Hall, London, 1989.

27. Faron Moller and Chris Tofts. A temporal calculus of communicating systems. Proceedings of CONCUR'90, 1990.

28. R. Paige and R.E. Tarjan. Three partition refinement algorithms. SIAM Journal of Computing, 16(6):973-989, December 1987.

29. G.D. Plotkin. A structural approach to operational semantics. Technical Report DAIMI-FN-19, Computer Science Department, Aarhus University, Aarhus, Denmark, 1981.

30. A. Pnueli. The temporal logic of programs. In $18^{\text {th }}$ Annual Symposium on Foundations of Computer Science, pages 46-57, Providence, Rhode Island, October/November 1977. IEEE.

31. Arnab Ray, Arne Skou, Rance Cleaveland, Scott Smolka, and Eugene Stark. Formal modeling and analysis of the control software for the cara infusion pump- draft report. CARA workshop, Monterrey, 2001.

32. S.C.Cheung and J.Kramer. Checking subsystem safety properties in compositional reachability analysis. 18th International Conference on Software Engineering, 1996. 